\title{
PROBLEMAS DE OTIMIZAÇÃO E MUDANÇA DE QUADROS EM UM TRABALHO COM PARTICIPANTES DE UM CURSO DE ENGENHARIA
}

\section{OPTIMIZATION PROBLEMS AND FRAMES CHANGING IN A WORK WITH PARTICIPANTS OF AN ENGINEERING COURSE}

\author{
Erique de Souza Siqueira \\ Universidade Anhanguera de São Paulo - UNIAN \\ eriquessiqueira@gmail.com \\ Maria Elisa Esteves Lopes Galvão \\ Universidade Anhanguera de São Paulo - UNIAN \\ elisa.gal.meg@gmail.com
}

\begin{abstract}
Resumo
$\mathrm{Na}$ experiência docente, constataram-se dificuldades dos estudantes dos semestres iniciais de um curso de engenharia para trabalhar com funções e suas representações. Teve-se como objetivo, nesta pesquisa, identificar a contribuição de uma intervenção baseada em um conjunto de problemas de otimização para a ampliação do conhecimento dos participantes sobre funções. A pesquisa foi orientada segundo o Design Experiment de Cobb et al, uma metodologia de natureza intervencionista cuja intenção é investigar as possibilidades de melhoria educacional, trazendo novas formas de aprendizagem. As ideias de Douady sobre mudanças de quadros forneceram o aporte teórico e buscou-se, com os problemas propostos, provocar tais mudanças por meio da resolução de problemas de otimização. Os participantes tiveram a oportunidade de analisar com mais profundidade e propriedade os dados e conceitos que lhes foram apresentados na educação básica e desenvolver uma visão sobre o entendimento e compreensão da utilização das funções para resolução de problemas de otimização.

Palavras-chave: Estudo de Funções; Resolução de Problemas; Otimização.
\end{abstract}

\begin{abstract}
In the teaching experience, difficulties to work with functions and their representations were found among students of the initial semesters of an engineering course. The objective of this research was to identify the contribution of an intervention based on a set of optimization problems to expand the participants' knowledge about functions. The research was guided by the Design Experiment by Cobb et al, a methodology of interventionist nature whose intention is to investigate the possibilities of educational improvement, bringing new forms of learning. Douady's ideas about frame changes provided the theoretical support and sought, with the proposed problems, to provoke such changes by solving optimization problems. The participants had the opportunity to analyze the data and concepts presented to them in basic education with more depth and propriety and to develop a view on understanding and understanding the use of functions to solve optimization problems.
\end{abstract}

Keywords: Mathematical Functions; Troubleshooting mathematical problems; Optimization. 


\section{INTRODUÇÃO}

Ao verificar que os alunos de curso superior de engenharia ainda não compreendiam as possibilidades para a exploração das propriedades das funções, na prática, e que muitos deles sequer sabiam que poderiam utilizar uma função adequada à resolução de um problema, a partir de uma análise introdutória, interpretação dos dados e construção de relações funcionais, nos propusemos a trabalhar sobre o tema na pesquisa de mestrado do primeiro autor, sob a orientação da segunda autora.

Preliminarmente, buscamos e examinamos alguns trabalhos que tratam de diferentes aspectos relacionados ao estudo e ao ensino e aprendizagem das funções. Oliveira (1997) contribuiu para o estudo do processo de ensino-aprendizagem das funções por meio de uma pesquisa realizada com professores de matemática. Baseou-se no "jogo de quadros" e na dialética "ferramenta-objeto" de Douady (1993), na preparação e aplicação de situações-problema. A pesquisadora destacou o fato de os livros didáticos influenciarem muito os professores, com a apresentação de problemas sobre funções de uma maneira pronta e acabada, não expondo o aluno a aplicações práticas para as funções. Zuffi (1999) nos apresentou um estudo histórico sobre o conceito de função, um panorama sobre a abordagem do ensino de funções por meio de diferentes métodos de aprendizagem, observações sobre a utilização de funções na resolução de problemas por meio de uma linguagem simples, sem muito formalismo, facilitando a aprendizagem. No artigo das autoras Galvão, Lobo da Costa e Prado (2017) é apresentada uma intervenção com um grupo de professores dando ênfase ao desenvolvimento de uma atitude investigativa na sala de aula, segundo Ponte (2003), com o auxílio de materiais de apoio tecnológico e material concreto. Dias (2017) nos traz um estudo apontando aspectos cognitivos e conceituais mobilizados quando os alunos estudam os pontos críticos de funções, levando em conta as estratégias utilizadas na construção da função. $O$ autor busca explicitar a compreensão e a construção dos conceitos de funções e seus pontos críticos pelos alunos e optou pelo Design Experiment para organizar metodologicamente o processo interventivo com alunos do curso de engenharia.

Apoiados nos resultados encontrados, organizamos uma intervenção com base em um conjunto de problemas relacionados a situações que possibilitam uma aplicação das funções lineares afins, quadráticas ou cúbicas, no contexto dos chamados problemas de otimização, que demandam o estudo da variação dessas funções para identificar os pontos 
de valor máximo ou mínimo. A busca dessas soluções ótimas, em situações simples, poderá propiciar que o estudante possa analisar com mais propriedade informações e/ ou conclusões sobre os dados que the são apresentados e ter uma atuação mais esclarecida e consciente na sociedade.

A pesquisa foi alicerçada teoricamente nos estudos de Douady e Perrin-Glorian (1989), sobre a resolução de problemas matemáticos por meio da mudança de quadros, provocada pelo professor, propondo o problema em um determinado quadro e levando o aluno a estudá-lo em um quadro diferente do proposto. A orientação metodológica se pautou pelo Design Experiment de Cobb et al (2003), e a análise dos dados tem caráter qualitativo. A coleta de dados foi realizada durante quatro encontros com a participação de estudantes dos semestres iniciais do curso de engenharia de uma instituição privada do interior do estado de São Paulo. Os participantes trabalharam em dupla ou individualmente. Foram selecionados, para análise, os protocolos, com as resoluções escritas de oito dos participantes que estiveram presentes em todos os encontros.

\section{REFERENCIAL TEÓRICO}

Adotamos como aporte teórico o trabalho de Régine Douady (1992), no qual se destaca a relevância das mudanças de quadro para o enriquecimento da atividade de resolução de um problema. Douady (1992) considera que a mudança de quadros é um caminho diferenciado para encontrar uma nova formulação para a resolução de um problema, podendo ser realizada de forma espontânea pelo aluno ou provocada pelo professor. É possível, por exemplo, mudar de um quadro de geometria euclidiana para a analítica, ou da geometria para as funções, a mudança dependerá da atividade proposta.

Douady (1986) relata que, para obter um cenário favorável à mudança de quadros, o professor deve levar em conta o fato de que o enunciado deve fazer sentido para o aluno. Destaca que o problema deve ser acessível do ponto de vista dos conhecimentos a serem mobilizados, de forma a possibilitar a discussão de estratégias para a resolução, priorizando ao menos dois quadros que sejam familiares aos estudantes. A mudança de quadros, segundo Douady (1986), é uma mudança que pode ser estimulada pelo professor, por meio de problemas propostos pertinentes, que levem os estudantes a uma situação de aprendizagem que avance nas fases de intervenção.

No processo de desenvolvimento da resolução dos problemas, a autora nos 
apresenta três fases. Na primeira fase, denominada fase de transferência e interpretação, os estudantes têm um problema estabelecido em um determinado quadro, e de acordo com os seus conhecimentos, a resolução do problema os conduz a trabalhar em um quadro diferente; é necessário que eles organizem os dados, encontrando formas de escolher as tentativas a serem realizadas. A mudança de quadro deve favorecer uma melhor interpretação da situação do problema pelo aluno. Temos a segunda fase, denominada fase de correspondências imperfeitas, na qual podem aparecer dificuldades devidas à falta de conhecimento dos estudantes; com as tentativas realizadas podem ou não chegar nas respostas, que em alguns casos podem ser, tentativamente, por eles manipuladas. Temos ainda a terceira fase, denominada fase de melhoria das correspondências e progresso no conhecimento, na qual se trabalha com a comunicação entre os quadros e com as suas práticas, na busca de soluções.

Douady (1986) afirma que, para se construir um ensino diferenciado, deve-se restabelecer um sentido para os instrumentos que os estudantes utilizam, apresentando esses objetos de uma forma institucional, de forma a se caracterizar uma diferente organização para o ensino.

Buscamos trabalhar com propostas de problemas que possibilitem aos alunos oportunidades de vivenciar o processo de resolução em diferentes quadros, de forma a aumentar a experiência na resolução dos problemas e na utilização de conhecimentos de maneira diferenciada daquela usualmente adotada na sala de aula.

Em nossa pesquisa, tratamos de um conjunto de problemas relacionados a situações que possibilitam a utilização das funções e o estudo da variação dessas funções nos quadros algébrico e gráfico, mudanças essas de quadros que devem ser realizadas por situações propostas pelo professor para que os alunos possam evoluir no conhecimento dos conceitos ao longo do processo de resolução de problemas.

\section{METODOLOGIA E PROCEDIMENTOS METODOLÓGICOS}

O "Design Experiment", de Cobb, Confrey, Di Sessa, Lehrer e Schauble (2003), suporte metodológico para a nossa pesquisa, tem sido uma metodologia muito utilizada em diversos campos relacionados à pesquisa educacional nos últimos anos, e, em especial, na Educação Matemática. Os autores nos apresentam três desafios. O primeiro desafio diz respeito à maneira como os professores conduzem as atividades no âmbito institucional, 
realizando as atividades no ambiente usual de suas aulas, e não em ambientes já preparados para um tipo específico de pesquisa, como por exemplo, um laboratório. O segundo desafio diz respeito à necessidade de um quadro interpretativo, que permita documentar a aprendizagem coletiva, mantendo tarefas desafiadoras promovidas na sala de aula e garantindo que os alunos tenham oportunidades para expressar o seu pensamento matemático nas discussões propostas. O terceiro desafio está relacionado ao objetivo de apoiar eventuais participações dos alunos às práticas fora do ambiente escolar.

Cobb et al (2003) indicam, no Design Experiment, formas de aprendizagem diferenciadas com uma tendência pragmática e uma orientação teórica. Experimentos realizados com o Design Experiment podem ser conduzidos para desenvolver novos conhecimentos, não apenas considerar os já existentes.

Com a utilização do Design Experiment, para orientar metodologicamente essa pesquisa, passamos a detalhar cada uma das fases previstas na sua organização, sendo elas:

- Fase Prospectiva: nessa fase, tratamos da análise das orientações curriculares institucionais propostas, que fazem parte dos itens do manual do professor, e dos livros didáticos, com relação às abordagens que foram sugeridas para o estudo de funções; da realização de um levantamento de pesquisas pertinentes que foram concluídas sobre o tema; e da identificação de diferentes tipos de funções associadas a aplicações e alguns exemplos que são apontados em livros didáticos;

- Fase Reflexiva: como previsto na metodologia, a pesquisa desenvolveu-se em etapas: inicialmente, nas aulas ministradas anteriormente à intervenção, foram observadas as dificuldades dos alunos na compreensão dos conteúdos ensinados; avaliamos, durante a intervenção, que, ao incluirmos exemplos práticos e situações problema próximas do real, a participação em sala de aula e a compreensão dos alunos aumentou consideravelmente;

- Fase Retrospectiva: correspondente à revisão analítica das atividades realizadas e seus registros, para produzir relatórios parciais ou conclusivos da pesquisa.

\section{DESENVOLVIMENTO DA PESQUISA}

Inicialmente, examinamos as orientações curriculares institucionais que fazem parte do manual do professor e dos livros didáticos apontados nas ementas das disciplinas de Matemática para a Engenharia. Consideramos as abordagens sugeridas para o estudo de funções e um levantamento das pesquisas pertinentes que foram concluídas sobre o tema, 
para elaborarmos a proposta de atividades. Convidamos os alunos para participar dos encontros e tivemos a adesão de um grupo de estudantes do $1^{\circ}$ ao $3^{\circ}$ semestre do ensino superior de uma faculdade privada do interior de São Paulo. Realizamos 4 (quatro) encontros para o trabalho com as atividades. Neste artigo, basearemos a análise nos protocolos escritos dos 8 alunos que participaram de todos os encontros.

As atividades propostas contemplaram conteúdos básicos sobre geometria e resolução de equações; buscamos possibilitar aos alunos a mobilização de seus conhecimentos sobre funções e uma melhor compreensão dos conceitos envolvidos na resolução dos problemas. Para tanto, os problemas foram propostos de forma a promover as mudanças de quadros, considerando os quadros geométrico, algébrico, gráfico e funcional, esse último associado à representação algébrico/funcional da situação-problema apresentada. No início de cada intervenção em sala de aula, foram apresentados as atividades e os objetivos de cada uma delas, de forma a orientar os participantes para o desenvolvimento do trabalho. Os alunos foram instruídos pelo professor a realizarem a resolução dos problemas propostos de acordo com o seu nível de conhecimento, esclarecidos no sentido de que não seriam considerados acertos nem erros e que não seriam atribuídas notas para efeito de avaliação em disciplinas regulares do curso.

No decorrer das atividades, os alunos foram motivados a discutirem as resoluções e considerarem diferentes alternativas de resolução, algumas vezes pelo professor, outras vezes por conta da própria atividade; eles eram incentivados a realizarem a mudança de quadros, de forma que pudessem ter uma outra visão da resolução da atividade proposta. Observamos que eles poderiam utilizar materiais de apoio tecnológico, como computador e calculadora, e materiais manipuláveis como o barbante e papel, para auxiliar na resolução das atividades. No término de cada sessão foram feitas as devidas explicações, e apresentadas resoluções, discutindo o que foi feito e o que poderia ter feito para encontrar a solução para o problema. Após a realização de cada encontro, os resultados obtidos nos protocolos escritos foram analisados, sob o ponto de vista qualitativo, para identificar os conhecimentos e os recursos mobilizados nas resoluções; após o primeiro encontro, as atividades escolhidas foram escolhidas de acordo com o grau de entendimento e resolução da atividade anterior. 


\section{ANÁLISE DE DADOS}

No primeiro encontro, o objetivo com o problema inicial (Figura 01), foi o de verificar o nível de compreensão dos alunos no que tange ao entendimento de geometria propriedades dos quadrados, triângulos retângulos e áreas - bem como os conhecimentos para a construção de uma função para descrever a área.

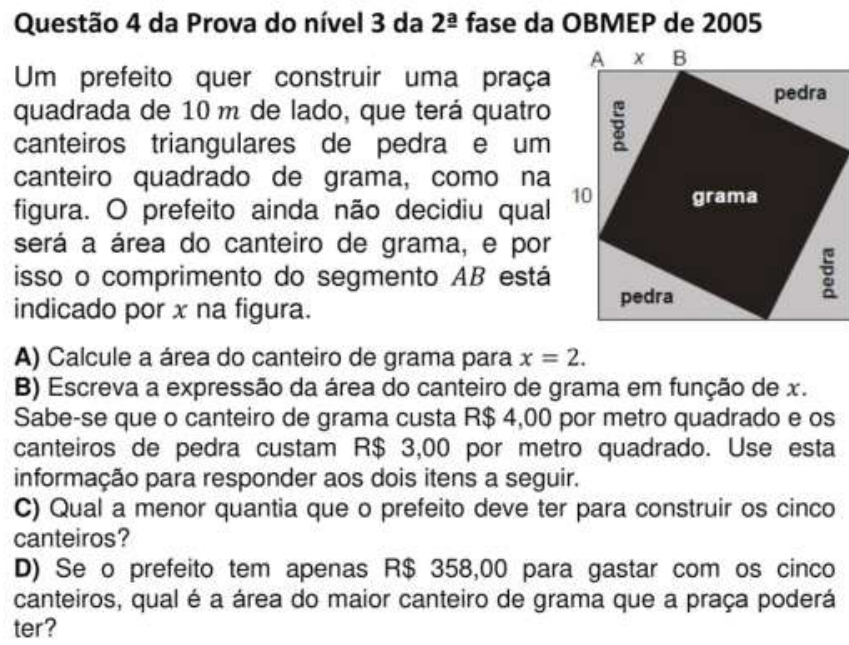

A) Calcule a área do canteiro de grama para $x=2$.

B) Escreva a expressão da área do canteiro de grama em função de $x$.

Sabe-se que o canteiro de grama custa $\mathrm{R} \$ 4,00$ por metro quadrado e os canteiros de pedra custam $\mathrm{R} \$ 3,00$ por metro quadrado. Use esta

informação para responder aos dois itens a seguir.

C) Qual a menor quantia que o prefeito deve ter para construir os cinco canteiros?

D) Se o prefeito tem apenas $R \$ 358,00$ para gastar com os cinco canteiros, qual é a área do maior canteiro de grama que a praça poderá ter?

Figura 01: Questão 4 da OBMEP 2005 - Nível 3 - 2ª Fase. Fonte: OBMEP - Nível $3-2^{\text {a }}$ Fase.

Também pretendíamos analisar aqui o conhecimento para se encontrar a área máxima e apresentar uma situação com valores estimados; uma vez obtida a função, há possibilidade de se encontrar valores máximos ou mínimos. Temos um problema apresentado no contexto geométrico, com informações em língua natural, cuja solução deve passar pelo quadro algébrico e, eventualmente, pelo gráfico.

Nessa resolução, ficou evidente que os alunos compreenderam bem a mudança do quadro geométrico para o numérico e que são capazes de encontrar a área a partir dos dados da figura, ou sejam passaram pela etapa da interpretação do problema e da transferência, sem dificuldades, mobilizando diferentes aspectos de seus conhecimentos. No item (a) os alunos usaram as medidas dos catetos e a fórmula da área do triângulo equilátero ou o teorema de Pitágoras para calcular a área solicitada; apenas dois dos participantes, que trabalharam em dupla, não concluíram o cálculo. No item (b) do problema, foi solicitada a mudança do quadro geométrico/numérico para o quadro funcional; ficou evidenciado que foi a mudança na qual os alunos tiveram mais facilidade para trabalhar. A função área foi descrita pelos alunos (Figura 02). 


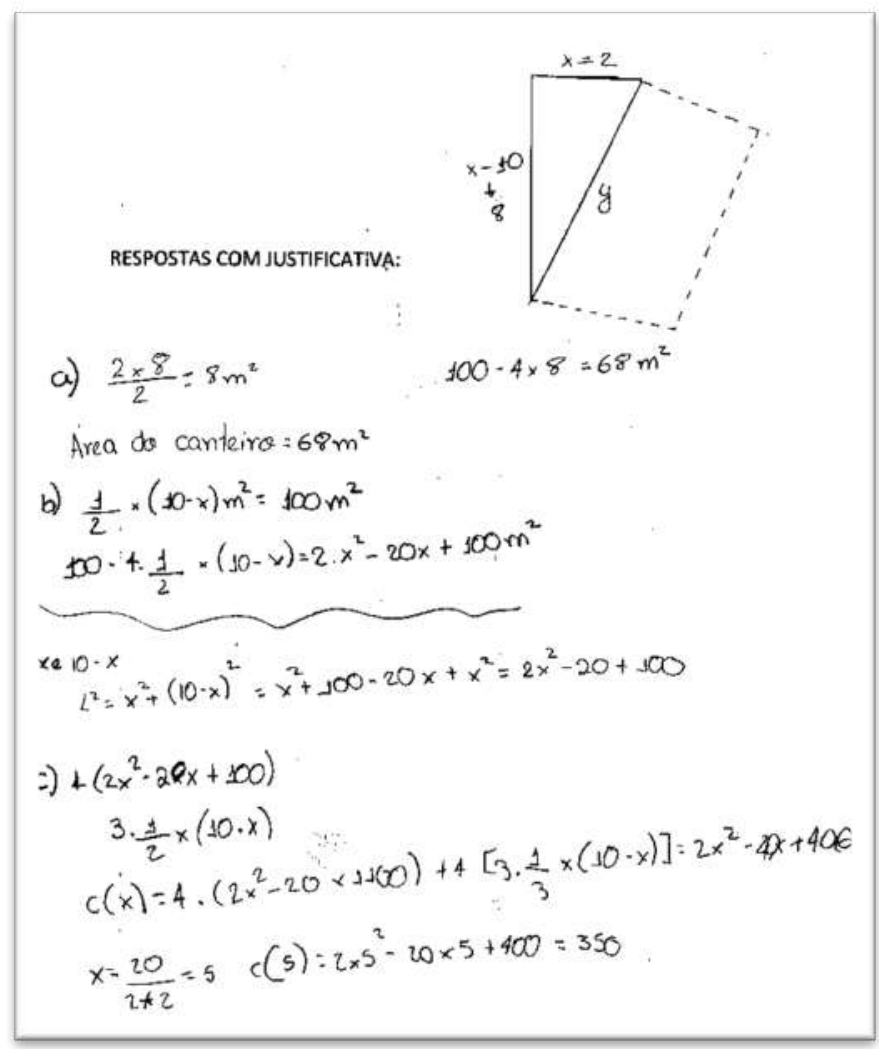

Figura 02: Resposta do problema do aluno "A6", da Atividade 1. Fonte: Protocolo do participante A6.

Para a resolução do item (c), os alunos desenvolveram os cálculos corretamente no quadro algébrico a partir da função obtida em (b); essa resolução aponta para o conhecimento do comportamento da função quadrática, identificando a primeira coordenada do vértice com o ponto médio das raízes. O gráfico da função não foi utilizado pelos participantes como suporte para solução. Nessa fase, denominada por Douady (1989) de fase de correspondências imperfeitas, a relação funcional provocada pela mudança de quadros foi encontrada e os elementos relacionados à resolução da função quadrática corretamente apontados; ao trabalhar com a mudança do quadro funcional para o quadro algébrico, os alunos demonstraram grande dificuldade e problemas com os cálculos. A fase de melhoria das correspondências e progresso no conhecimento, na qual a resolução, no quadro algébrico, seu retorno para a interpretação dos resultados e o item (d) foram prejudicados e se constituíram o grande desafio da atividade; os alunos não tiveram o mesmo entendimento do item anterior. No item (d) nenhum aluno encontrou a resposta corretamente, deixaram em branco; o aluno "A5" (Figura 03) explicitou a dificuldade encontrada nessa alternativa. 


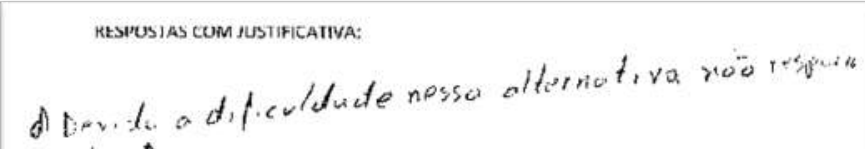

$$
\begin{aligned}
& \text { a tetos D }
\end{aligned}
$$

Figura 03: Resposta do aluno "A5", da Atividade 1 - Item D.

Fonte: Protocolo do participante A5

Iniciamos o segundo encontro com um problema que recuperava algumas ideias da atividade anterior (Figura 04), para investigar como os alunos compreendem um problema sem valores numéricos, como raciocinam para encontrar uma solução e que estratégia iriam mobilizar para resolver a questão. Podemos considerar essa questão proposta, do ponto de vista de seu objetivo, uma extensão da fase de melhoria das correspondências e progresso no conhecimento da questão anterior, uma vez que provoca uma interpretação que mobiliza aspectos do quadro geométrico explorado inicialmente.

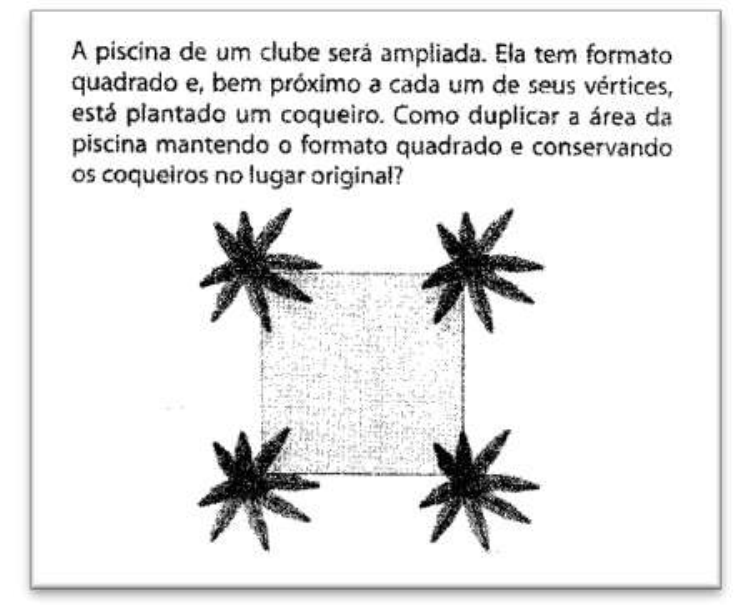

Figura 04: Problema sobre a ampliação de uma piscina.

Fonte: Matemática, $8^{\circ}$ ano, Projeto Araribá. Ed. Moderna (2013).

A resolução natural, na fase de interpretação e transferência, permanece no quadro geométrico no qual é formulado o problema, e a estratégia a ser adotada remete ao caminho inverso da resolução da primeira atividade. Ocorreu a transferência, sem dificuldades, de elementos da questão anterior. Constatamos, na fase de correspondências que, de forma simples, os alunos resolveram a atividade simplesmente girando e aumentando a figura (Figura 05). Um dos alunos explicitou sua resposta apontando o quadrado de área maior e observando que o vértice do novo quadrado gira $45^{\circ}$ em relação à direção do vértice do quadrado menor, rotação essa cujo centro é o centro do quadrado original, mantendo os coqueiros no mesmo local. 


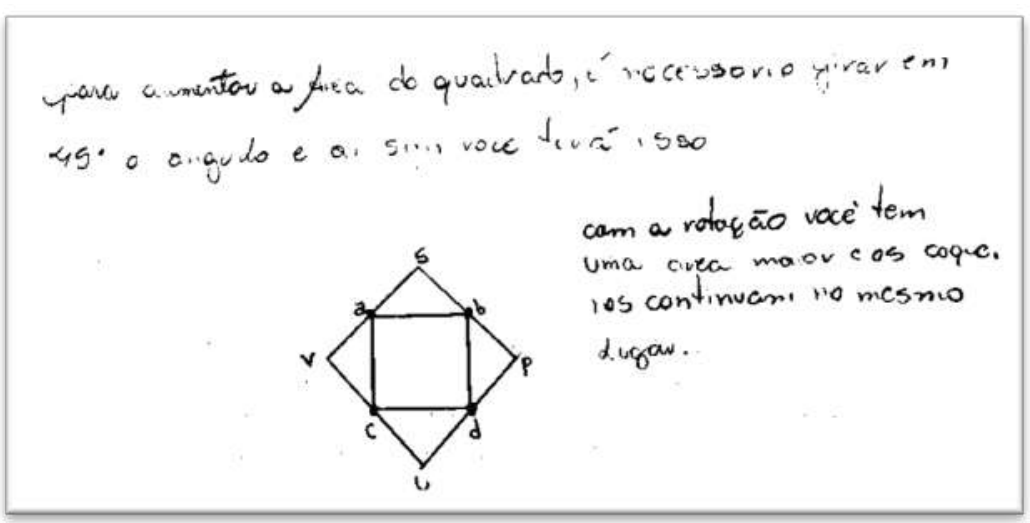

Figura 05: Resposta do aluno “A2", da Atividade 2.

Fonte: Protocolo do participante A2.

Como melhoria das correspondências e progresso no conhecimento, chamam a atenção as referências às rotações, como parte dos conhecimentos mobilizados em algumas das resoluções apresentadas.

No terceiro encontro, propusemos dois problemas. O primeiro problema (Figura 06) visava verificar como os participantes interpretariam a questão, quais procedimentos utilizariam para resolver um problema de otimização, se conseguiam visualizar que, ao alterar algum lado do retângulo, influenciariam diretamente no valor da área.

1. (Dante, 2004). Deseja-se construir uma casa térrea de forma retangular. O retângulo onde a casa será construída tem $80 \mathrm{~m}$ de perímetro. Calcule as dimensões desse retângulo sabendo que a área de sua região interior deve ser a maior possível.

Figura 06: Problema sobre a área máxima Atividade 3 - Problema 1.

Fonte: Acervo pessoal.

Os alunos conseguiram, de uma forma geral, na fase de interpretação e transferência, fazer as passagens do quadro geométrico para o quadro algébrico ou algébrico/funcional e chegar à expressão algébrica para a função; conseguiram interpretar o enunciado do problema, usar que a somadas medidas dos lados é igual ao perímetro informado no enunciado. Os alunos também compreenderam que dependiam da fórmula da área para encontrar as dimensões do lado do retângulo cuja área é máxima, o que podemos considerar já um primeiro passo na fase das correspondências imperfeitas.

Na continuidade dessa fase, verificamos na resolução apresentada que o aluno "A8" (Figura 07) utilizou a compreensão do quadro geométrico para auxiliar na resolução do problema; além disso, fez corretamente as mudanças para os quadros quadro algébrico e 
algébrico/funcional. Diante da função gerada tivemos protocolos em que a resolução apresentada recorreu à fórmula do vértice $x_{v}=-\frac{b}{2 a}$, da parábola que é o gráfico da função quadrática; alguns alunos derivaram a função e igualaram a zero para encontrar o valor de " $x$ ", porém não fizeram o estudo da variação da função. Em ambos os casos chegaram à solução correta. Novamente, um dos participantes resolveu tabelando as possibilidades de soluções inteiras. Observamos que diferentes conhecimentos e correspondências foram mobilizados nas resoluções apresentadas, destacando mais uma vez a ausência explícita do recurso ao gráfico da função envolvida.

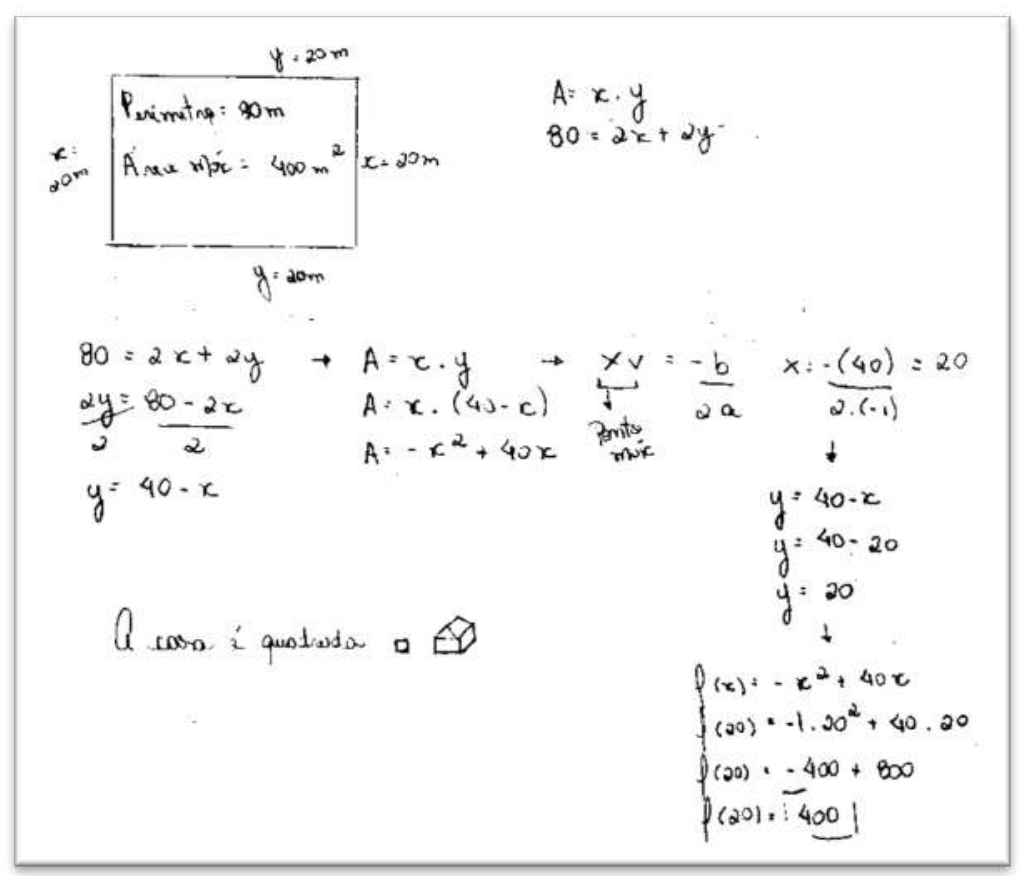

Figura 07: Resposta do aluno “A8”, da Atividade 3 - Problema 1. Fonte: Protocolo do participante A8.

Com o segundo problema proposto, o objetivo foi verificar se os alunos seriam capazes de encontrar uma função para determinar o volume máximo de uma caixa. Esse problema foi incluído pois, diferentemente dos problemas anteriores, se obtém uma equação do terceiro grau, e com isso encontrar um valor de "x" ideal para a solução do problema requer a mobilização de recursos que foram ou estavam sendo explorados no Cálculo Diferencial. Novamente, descreve-se um quadro geométrico para que o aluno encontre a função adequada para a resolução (Figura 08). 
2. Uma caixa sem tampa deve ser construída de um pedaço retangular de papeläo com dimensões $8 \mathrm{dm}$ por $5 \mathrm{dm}$. Para isso, deve se retirar quadrados de lado $x$ de cada canto e depois dobrar, conforme mostra a figura. a) Expresse o volume $v$ da caixa como uma função de x. b) Determine o volume máximo dessa caixa.
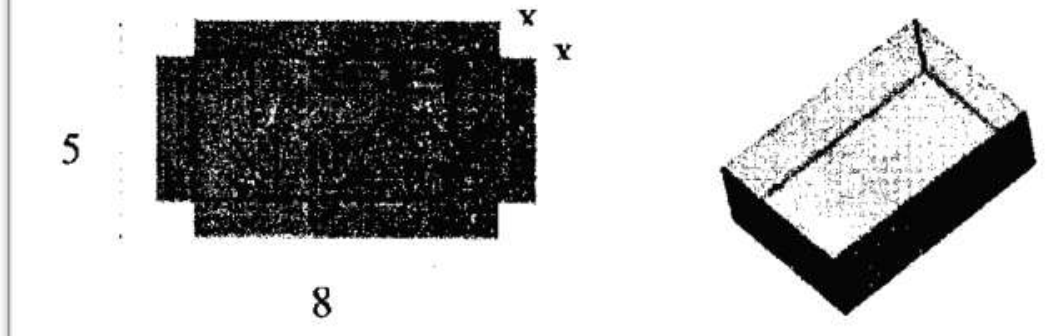

Figura 08: Problema sobre a área máxima Atividade 3 - Problema 2.

Fonte: Acervo pessoal.

Novamente, na fase de interpretação e transferência, os participantes compreenderam bem a passagem do enunciado para o quadro geométrico/algébrico, e posteriormente para o quadro algébrico/funcional, escrevendo corretamente a função do volume; no entanto, não se ativeram ao intervalo de variação da função, associado às possibilidades de comprimento do corte. Na sequência, estabelecidas as correspondências para determinar o ponto de mínimo, alguns derivaram a função igualando a 0 (zero) para encontrar a raiz da função quadrática; alguns alunos conseguiram encontrar o valor correto de "x", porém não deram continuidade à resolução do problema para encontrar o volume máximo.

Verificamos, no protocolo exibido, que o aluno A10, executou bem a passagem para o quadro geométrico/algébrico e para o quadro algébrico/ funcional, chegando corretamente à função correspondente ao volume, na primeira fase da resolução; também constamos o cálculo correto da primeira derivada, e uma simplificação para encontrar as raízes da função quadrática, parte da fase das correspondências imperfeitas. Esse participante encontrou o valor correto de " $x$ ", porém não deu andamento à resolução e não fez o estudo da variação da função (Figura 09), ou seja, as correspondências foram estabelecidas, os conhecimentos sobre a variação do sinal das expressões algébricas comprometeram a última etapa da resolução. 


$$
\begin{aligned}
& V=x(8-2 x)(5-2 x) \Rightarrow 4 x^{3}-26 x^{2}+40 x \\
& V^{\prime}=12 x^{2}-52 x+40=0 \\
& 3 x^{2}-13 x+10=0 \\
& \Delta=169-120=49 \\
& \sqrt{\Delta}: \sqrt{49}=7 \\
& \pi:(13-7): 6=1 \mathrm{~cm} .
\end{aligned}
$$

Figura 09: Resposta do aluno “A7”, da Atividade 3 - Problema 2.

Fonte: Protocolo do participante A7.

No quarto encontro, abordamos dois problemas. Com o primeiro problema (Figura10) tivemos como objetivo principal verificar se os alunos sabem relacionar elementos do gráfico de uma parábola à correspondente função do segundo grau, ou seja, começamos pelo quadro gráfico. Por meio da leitura do gráfico, o aluno precisaria identificar quais são os valores correspondentes às coordenadas dos pontos associados às alturas solicitadas no enunciado.

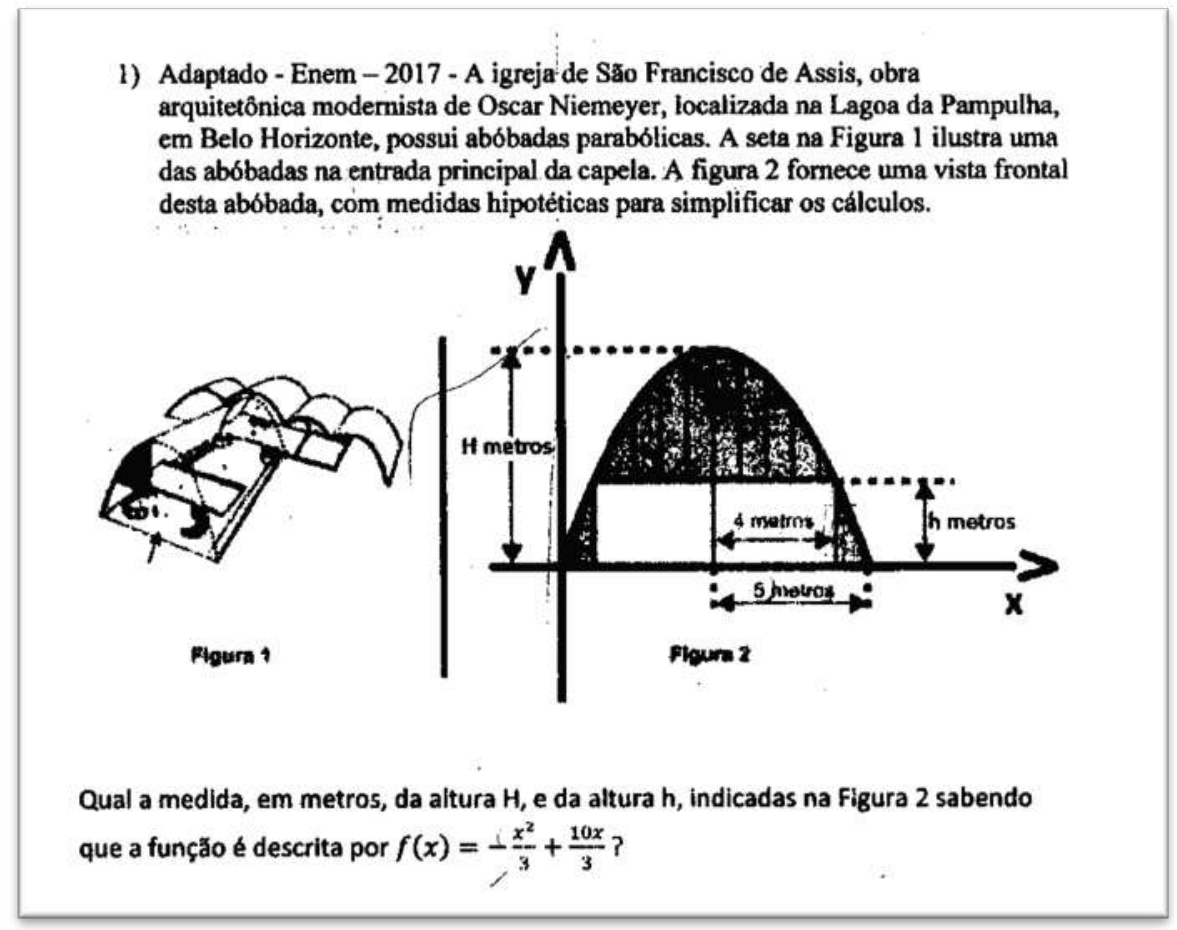

Figura 10: Problema - cálculo de altura - Atividade 3 - Problema 2. Fonte: Acervo pessoal.

Verificamos, no protocolo exibido na Figura 11, uma solução na qual o aluno conseguiu interpretar corretamente a mudança de quadros, interpretando o gráfico de forma 
a substituir os valores de " $x$ ", e utilizar a expressão da função, utilizando a interpretação correta do quadro funcional na primeira etapa da resolução.

$$
\begin{array}{ll}
f(x)=\frac{-5^{2}}{3}+\frac{10 \cdot 5}{3} & f(x)=-\frac{9^{2}}{3}+\frac{10 \cdot(9)}{3} \\
H=\frac{-25}{3}+\frac{50}{3} & h=\frac{-81}{3}+\frac{90}{3} \\
H=\frac{25}{3} & h=\frac{9}{3} \\
H=8,3 \mathrm{~m} & h=3 \mathrm{~m}
\end{array}
$$

Figura 11: Resposta do aluno “A4”, da Atividade 4 - Problema 1.

Fonte: Protocolo do participante A4.

Constatamos uma dificuldade encontrada pelos participantes para completar essa passagem: identificar o ponto " $x$ ”, para encontrar as alturas desejadas. Nessa mudança de quadros gráfico/funcional precisaríamos apenas substituir um determinado valor de " $x$ " encontrado no gráfico da função, para assim encontrarmos o valor de " $f(x)$ ". Um dos participantes, o aluno A2, optou por iniciar a resolução pelo quadro funcional, usando a fatoração da função do segundo grau, entretanto considerou raízes 5 e -5 e não 0 e 10 ; porém o aluno acabou se perdendo na resolução, errando o sinal do produto notável, e não conseguiu fazer a mudança de quadro corretamente, conforme (Figura 12); o aluno determinou o valor correto para a altura $\mathrm{H}$, solicitada no problema, utilizando a expressão errada para a função. As correspondências a serem estabelecidas entre os elementos do domínio e as alturas a serem encontradas, na segunda fase da resolução do problema, se constituíram uma dificuldade, em geral, que comprometeu a fase final da resolução do problema.

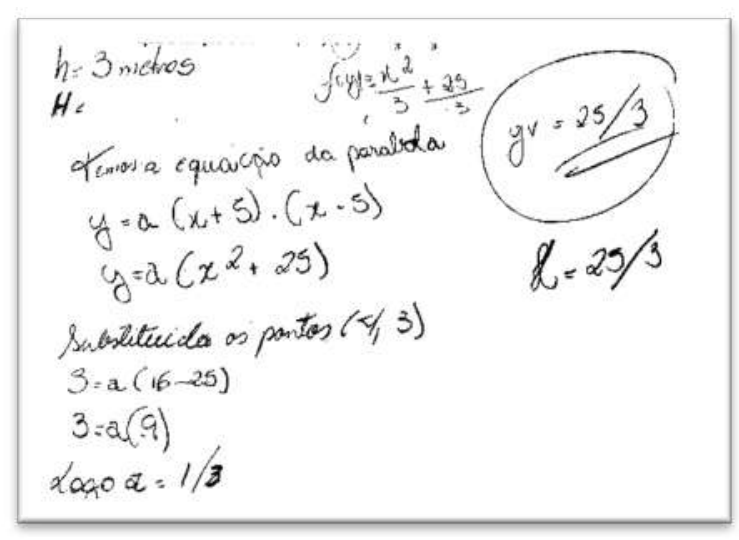

Figura 12: Resposta do aluno “A2”, da Atividade 4 - Problema 1.

Fonte: Protocolo do participante A2. 
O segundo problema apresentava semelhanças com o problema inicial do terceiro encontro, utilizando apenas 3 lados do retângulo. Novamente, passa-se de uma descrição no quadro geométrico para o quadro algébrico correspondente, na fase de interpretação e transferência. A proposta visava verificar se eles eram capazes de identificar e explicitar como, com as alterações das dimensões do comprimento e das larguras, a área seria alterada (Figura 13).

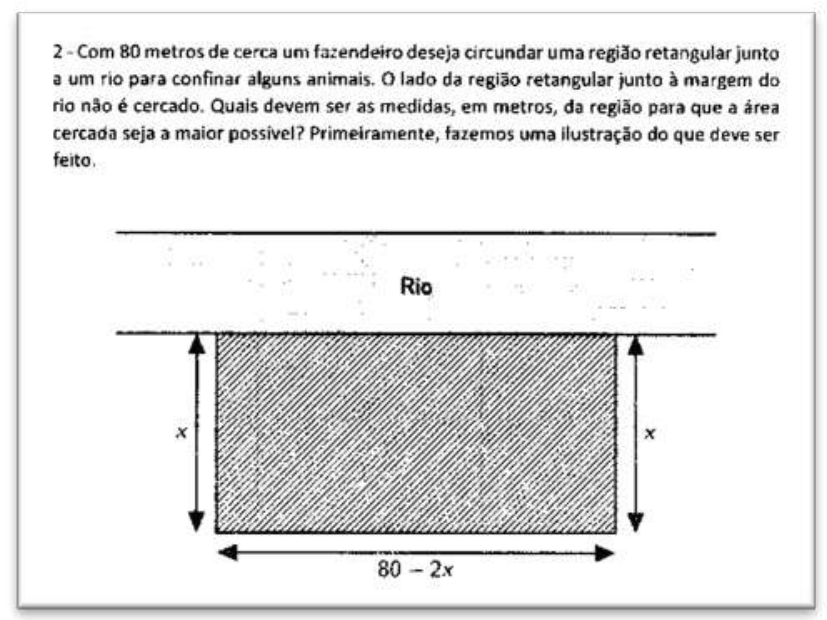

Figura 13: Problema sobre a área máxima Atividade 4 - Problema 2.

Fonte: Acervo pessoal.

Dentre os alunos que desenvolveram corretamente a resolução do problema, destacamos na Figura 14 a resolução na qual o aluno A4, passa corretamente do quadro geométrico para o quadro algébrico/funcional, na primeira fase, encontrando o valor do vértice para obter o valor de " $\mathrm{x}$ " correspondente à solução; posteriormente o aluno substitui corretamente na expressão da função o valor de "x", passando para o quadro algébrico e encontrando o valor da área com o auxílio da função, obtendo o ponto máximo, mobilizando as correspondências necessárias para o desenvolvimento da segunda fase e posteriormente, os conhecimentos necessários sobre a variação da função quadrática para a conclusão da última fase da resolução. 


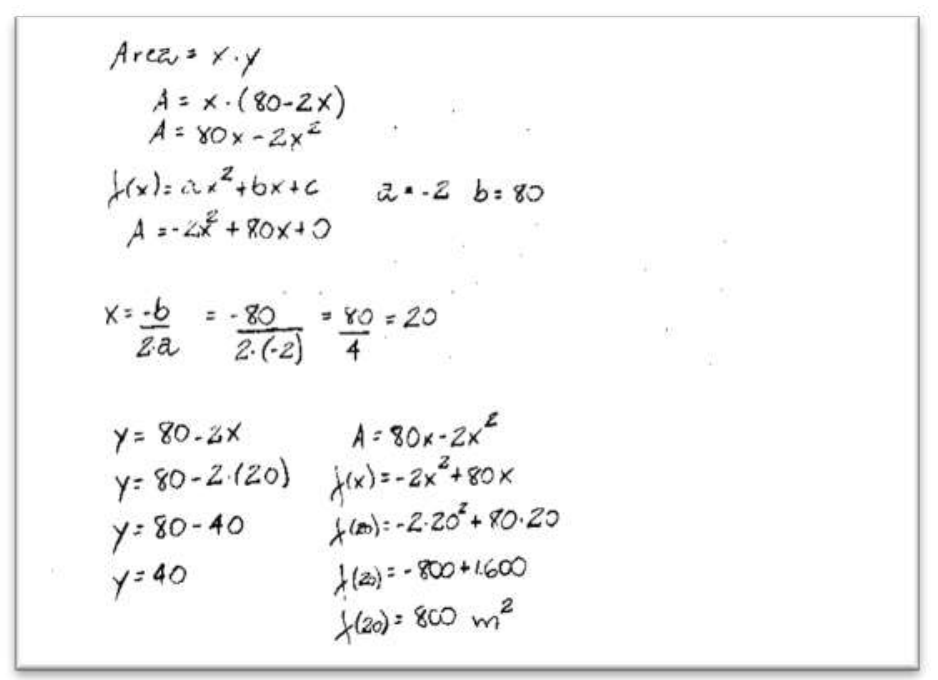

Figura 14: Resposta do aluno "A4", da Atividade 4 - Problema 2. Fonte: Protocolo do participante A4.

No contexto geral dos resultados, os participantes apresentaram bons resultados em uma parte significativa dos problemas propostos; conseguiram de modo geral, trabalhar com a mudança de quadros, tendo uma maior dificuldade na mudança para os quadros algébrico/funcional conforme quadro 01, o que nos leva a afirmar que, em geral, a fase da interpretação e transferência foi concluída com êxito. Por outro lado, se viram muito motivados e empenhados nas resoluções dos problemas; a grande dificuldade apresentada pelos participantes, a partir das correspondências incompletas, em parte estabelecidas, em quase todos os problemas, foi com os procedimentos algébricos, essenciais para levar a bom termo resolução dos problemas.

\begin{tabular}{|c|l|c|c|c|c|c|c|c|c|c|}
\hline Atividade & \multicolumn{1}{|c|}{ Quadro } & A1 & A2 & A3 & A4 & A5 & A6 & A7 & A8 & Acerto \\
\hline 1 & quadro numérico/ algébrico & $\operatorname{sim}$ & $\operatorname{sim}$ & $\operatorname{sim}$ & $\operatorname{sim}$ & $\operatorname{sim}$ & Sim & $\operatorname{sim}$ & $\operatorname{sim}$ & $100 \%$ \\
\hline 1 & quadro algébrico/ funcional & $\operatorname{sim}$ & $\operatorname{sim}$ & $\operatorname{sim}$ & $\operatorname{sim}$ & $\operatorname{sim}$ & Sim & $\operatorname{sim}$ & $\operatorname{sim}$ & $100 \%$ \\
\hline 1 & quadro geométrico/ algébrico & $\operatorname{sim}$ & $\operatorname{sim}$ & $\operatorname{sim}$ & $\operatorname{sim}$ & $\operatorname{sim}$ & Sim & $\operatorname{sim}$ & não & $88 \%$ \\
\hline 1 & Quadro algébrico/ gráfico & não & $\operatorname{sim}$ & não & não & não & Não & $\operatorname{sim}$ & não & $25 \%$ \\
\hline 2 & quadro geométrico & $\operatorname{sim}$ & $\operatorname{sim}$ & $\operatorname{sim}$ & $\operatorname{sim}$ & $\operatorname{sim}$ & Sim & $\operatorname{sim}$ & $\operatorname{sim}$ & $100 \%$ \\
\hline 3.1 & quadro geométrico & $\operatorname{sim}$ & não & $\operatorname{sim}$ & não & $\operatorname{sim}$ & Sim & não & $\operatorname{sim}$ & $63 \%$ \\
\hline 3.1 & quadro algébrico & $\operatorname{sim}$ & $\operatorname{sim}$ & $\operatorname{sim}$ & $\operatorname{sim}$ & não & Não & $\operatorname{sim}$ & $\operatorname{sim}$ & $75 \%$ \\
\hline 3.1 & quadro funcional & $\operatorname{sim}$ & $\operatorname{sim}$ & $\operatorname{sim}$ & $\operatorname{sim}$ & $\operatorname{sim}$ & Não & $\operatorname{sim}$ & $\operatorname{sim}$ & $88 \%$ \\
\hline 3.2 & quadro geométrico/ algébrico & $\operatorname{sim}$ & $\operatorname{sim}$ & $\operatorname{sim}$ & $\operatorname{sim}$ & não & Não & $\operatorname{sim}$ & $\operatorname{sim}$ & $88 \%$ \\
\hline 3.2 & quadro algébrico/ funcional & $\operatorname{sim}$ & $\operatorname{sim}$ & $\operatorname{sim}$ & $\operatorname{sim}$ & não & Não & $\operatorname{sim}$ & $\operatorname{sim}$ & $75 \%$ \\
\hline 4.1 & quadro gráfico/ funcional & $\operatorname{sim}$ & $\operatorname{sim}$ & $\operatorname{sim}$ & $\operatorname{sim}$ & $\operatorname{sim}$ & Sim & não & $\operatorname{sim}$ & $88 \%$ \\
\hline 4.2 & quadro algébrico & $\operatorname{sim}$ & $\operatorname{sim}$ & $\operatorname{sim}$ & $\operatorname{sim}$ & $\operatorname{sim}$ & Sim & $\operatorname{sim}$ & $\operatorname{sim}$ & $100 \%$ \\
\hline 4.2 & quadro funcional & não & não & não & $\operatorname{sim}$ & não & Não & não & $\operatorname{sim}$ & $25 \%$ \\
\hline
\end{tabular}

Quadro 01: Percentagem de acertos por quadro x participantes.

Fonte: Elaborado pelo autor. 
Nas atividades realizadas conseguimos fazer com que os participantes compreendessem a importância da mudança de quadros e do trabalho com problemas práticos. Conseguiram vivenciar como a matemática pode auxiliar a resolução de problemas reais, mobilizar diferentes conhecimentos na resolução dos problemas, e consideramos que a mudança de quadros foi uma ferramenta importante no processo de resolução.

Verificamos as dificuldades encontradas na busca de solução para problemas de otimização relacionados a diferentes contextos foram devidas, principalmente, ao preparo dos alunos no ensino médio ou ainda no ensino fundamental em relação aos cálculos algébricos. Outras dificuldades apresentadas durante as sessões, em muitos momentos, se deram devido ao perfil geral dos alunos que participaram das intervenções; muitos deles, ingressantes na faculdade, estavam há mais de 5 anos sem estudar, grande parte deles vem do ensino público e, frequentemente, não lembravam de operações básicas da matemática ou não tiveram oportunidade de conviver e amadurecer adequadamente os conteúdos ainda nessa fase de escolaridade.

Durante as atividades os alunos demonstraram grande interesse em realizá-las, os desafios aqui apresentados trouxeram para os alunos uma grande motivação e provocou um forte envolvimento do grupo. De certa forma eles demostraram facilidade nas mudanças de quadro. No geral observamos que a passagem do quadro geométrico para outros quadros foi a mais utilizada; no quadro gráfico, demonstraram maior dificuldade e não fizeram uso do mesmo em qualquer momento para iniciar uma atividade, salvo no problema que partia dessa representação, no qual o aluno era obrigado a analisar primeiramente esse quadro.

Analisamos e constatamos que contribuição relevante da mudança de quadros foi propiciar a oportunidade de integrar diferentes conhecimentos na atividade de resolução de problemas. Acreditamos que trouxemos uma contribuição para ampliar o conhecimento dos participantes no estudo de funções no âmbito da resolução de problemas práticos, pois eles conseguiram compreender e desenvolver estratégias para organizar as resoluções. 


\section{CONSIDERAÇÕES FINAIS}

Durante a realização dessa pesquisa foram analisados diversos aspectos relativos à compreensão dos alunos participantes quando expostos a problemas que possibilitam o estudo do comportamento das funções, dentre outros, aqueles relacionados à otimização.

Optamos nesse estudo para analisar como eles trabalhariam com a mudança de quadros e como a mudança de quadros pode contribuir para ampliar o conhecimento dos alunos no que tange ao estudo de funções, no contexto de resolução de problemas práticos de otimização.

As atividades elaboradas e propostas na nossa pesquisa demonstraram tais poderes da mudança de quadros, que mesmo diante de seus limites, podem trazer inúmeros benefícios para professores e estudantes.

As narrativas dos alunos durante as sessões foram significativas, tendo um papel fundamental em todo o processo desenvolvido, no que tange ao conceito de função. Em todos os momentos os alunos explicitavam suas ideias, os seus pensamentos e seus raciocínios, debatiam entre si buscando argumentos para justificar suas respostas.

Acreditamos que, conforme os Parâmetros Curriculares Nacionais (PCN, 1998), sobre a Resolução de Problemas: “o conhecimento matemático ganha significado quando os alunos têm situações desafiadoras para resolver e trabalham para desenvolver estratégias de resolução" (PCN, 1998, p. 40). A resolução de problemas pode ser uma oportunidade para percorrer caminhos diferentes na obtenção de uma solução, estimular o aluno para que ele questione sua resposta para um problema, formule diferentes respostas; é uma oportunidade para demonstrar aos alunos que eles podem mudar a forma não só de enxergar a matemática, mas a forma de gostar da matemática.

A mudança de quadros e aplicação de problemas práticos, aliada à metodologia do Design Experiment adotada nessa dissertação contribuíram para o bom desenvolvimento da pesquisa realizada, pois quando fazíamos um encontro, analisávamos os resultados e a partir disso, preparávamos o material para o próximo encontro. Durante o desenvolvimento das atividades sempre buscamos desafiar o aluno a progredir e criar formas novas de buscar o conhecimento. 


\section{REFERÊNCIAS}

COBB, P.; CONFREY, J.; DISESSA, A.; LEHRER, R.; SHAUBLE, L. Design Experiments in Educational Research. In: Educational Researcher, v. 32, n. 1, Jan/ Fev, 2003, p. 9-13.

DIAS, R. R. Aspectos cognitivos e conceituais mobilizados na resolução de problemas de otimização por estudantes de engenharia. Tese de Doutorado em Educação Matemática, Universidade Anhanguera de São Paulo, São Paulo, 2017.

DOUADY, R. Recherches em Didactiques des Mathématiques. 7, 1986, p. 5-31.

DOUADY, R. Repères - IREM. No 6 - Janvier 1992.

DOUADY, R., PERRIN-GLORIAN, D'apprentissage du Concept D'aire de Surface Plane, Educational Studies in Mathematics,Vol. 20(4),1989. 387-424.

GALVÃO, M. E. E. L.; LOBO DA COSTA, N. M.; PRADO, M. E. B. B. Construção de funções a partir de problemas geométricos: Uma abordagem investigativa. REnCiMa, p. 39-57, 2017.

OLIVEIRA, N. Conceito de função: uma abordagem do processo ensinoaprendizagem. Dissertação de Mestrado em Ensino da Matemática, Pontifícia Universidade Católica de São Paulo, São Paulo, 1997.

PCN, Parâmetros Curriculares Nacionais ( $5^{\mathrm{a}}$ a $8^{\mathrm{a}}$ Séries), Secretaria de Educação Fundamental. Brasília: MEC / SEF, 1998.

ZUFFI, E. M. O tema funções e a linguagem matemática de professores do ensino médio - por uma aprendizagem de significados. Tese de doutorado da Universidade de São Paulo, São Paulo, 1999.

Submetido em 03 de agosto de 2020. Aprovado em 10 de fevereiro de 2021. 